\title{
Multiuser Detection Schemes for Multicarrier Four-node Wireless Systems
}

\author{
Mohamed Marey, Senior Member*, IEEE, Khaled Almustafa* and Hala Mostafa \\ College of Engineering, Prince Sultan University, Riyadh, KSA* \\ Faculty of Electronic Engineering, Menoufia University, Menouf, Egypt $t^{t}$ \\ Email \{mfmmarey@psu.edu.sa*,kalmustafa@psu.edu.sa*,h.mostafa@mun.ca $\}$
}

\begin{abstract}
This paper proposes two novel data detectors for an uplink concatenated system which includes multiuser orthogonal frequency division multiplexing (OFDM) and four-node cooperative transmission. The structure of the received signal is exploited to design the first detector using maximum likelihood $(M L)$ principle. The second proposed detector utilizes the principle of sliding window to enhance the processing time of the first one. The results from Monte Carlo simulations show us that both detectors are effective; however, the bit error rate performance the first detector sightly outperforms that of the second one.
\end{abstract}

Keywords: $M L$, SIC, Data detection

\section{Introduction}

Recently multiuser OFDM modulation has been incorporated into several wireless applications such as cellular systems and wireless local area systems [8]. It has shown to be efficient in treating multiple access interference and multipath environments while maintaining high spectral efficiency. In addition, its architecture is able to provide different quality of service per user by employing dynamic subcarrier assignment.

Cooperative technology has been proposed to improve error rate performance and capacity of communication systems through forming a virtual antenna array with the available antenna terminals [9]. The core idea is that the nodes in a communication system cooperate by forwarding their data to achieve spatial diversity. However, this technology faces significant reduction in the spectral efficiency due to the half-duplex constraint at the relays. Two-path cooperative (TPC) transmission protocols have been introduced to enhance such reduction [5,7], where two relays alternatively forward data of the transmitted node to destination. Generally speaking, the previous work on TPC can be categorized into two groups of studies. A group has concentrated on the information theory issues such as capacity-outage and diversity-multiplexing tradeoff analysis $([1,4])$ while the second one is devoted to data detection algorithms $[2,3,6]$. The previously mentioned works of the second group were considered a single user with single carrier transmission.

In this work, we develop an TPC transmission protocol for a multiuser OFDM system using decode-and-forward (DF) relaying scheme. In addition, we propose two data detectors at the destination. The undesirable interference signal at the destination, which results from continuous forwarding messages, is utilized to design both detectors while maintaining spatial diversity.

The remainder of the paper is structured as follows: System model and formulation are presented in Section 2. The proposed two detectors at the destination are presented in Sections 3, and 4. The performance evaluation is in Section 5 and the conclusion is in Section 6. 


\section{System Description}

We assume an orthogonal frequency division multiple access system employing $U$ users, $Q$ subcarriers and two relays. A group of non-overlapping subcarriers $S^{(u)}=2$

Table 1. Transmission Time-lines of the TPC Multiuser OFDM System

\begin{tabular}{|c|c|c|c|c|c|c|}
\hline Block number & 1 & 2 & 3 & 4 & 5 & $\ldots$ \\
\hline Users & Transmit & Transmit & Transmit & Transmit & Transmit & $\ldots$ \\
\hline First relay & Receive & Transmit & Receive & Transmit & Receive & $\ldots$ \\
\hline Second relay & & Receive & Transmit & Receive & Transmit & $\ldots$ \\
\hline Destination & Receive & Receive & Receive & Receive & Receive & $\ldots$ \\
\hline
\end{tabular}

$\left\{s_{1}^{(u)}, s_{2}^{(u)}, \cdots, s_{j}^{(u)}\right\}$ are assigned to the $u$ th user, where $J=Q / U$ is the number of subcarrier per user. The transmitter sends frames to a destination; each frame has $B$ blocks each of which consists of $J$ symbols. The transmission time-lines of the proposed system is shown in Table 1. One can observe that the loss of spectral efficiency is recovered by allowing each user continues sending his $B$ data blocks per frame to the relays and destination.

At the destination, the received data symbols on the subcarrier $s \in S^{(u)}$ of the $b$ th and $(b+1)$ th blocks of the $u$ th user can be given as

$$
\begin{array}{r}
z_{b}^{(u)}(s)=a_{b}^{(u)}(s) g_{X}^{(u, b)}(s)+a_{b-1}^{(u)}(s) g_{T_{a}}^{(b)}(s) \lambda_{T_{0}}^{(u)}(b-1)+n_{b}^{(u)}(s), \\
z_{b+1}^{(u)}(s)=a_{b+1}^{(u)}(s) g_{X}^{(u, b)}(s)+a_{b}^{(u)}(s) g_{T_{l}}^{(b)}(s) \lambda_{T_{f}}^{(u)}(b)+n_{b+1}^{(u)}(s),
\end{array}
$$

where $a_{b}{ }^{u}(s)$ is the frequency domain data symbol sent by user $u$ on the subcarrier $s$ at the block $b, g_{x}{ }_{x}^{u, b)}(s)$ is the channel coefficient corresponding to the subcarrier $s$ between user $u$ and the destination at the block $\left.b, g_{T}{ }^{b}\right)(s)$ is the channel coefficient corresponding to the subcarrier $s$ between relay $T_{o}$ and the destination, $n_{b}{ }_{b}^{u)}(s)$ is the noise contribution, and (u)

$\lambda_{T^{l}}(b)$ is a coefficient having a value one if the block $b$ is correctly detected at the relay $T_{o}$ and zero otherwise. In this work, we assume that channel information is available at the destination. The detailed received signal structure at the destination is shown in figure 1.
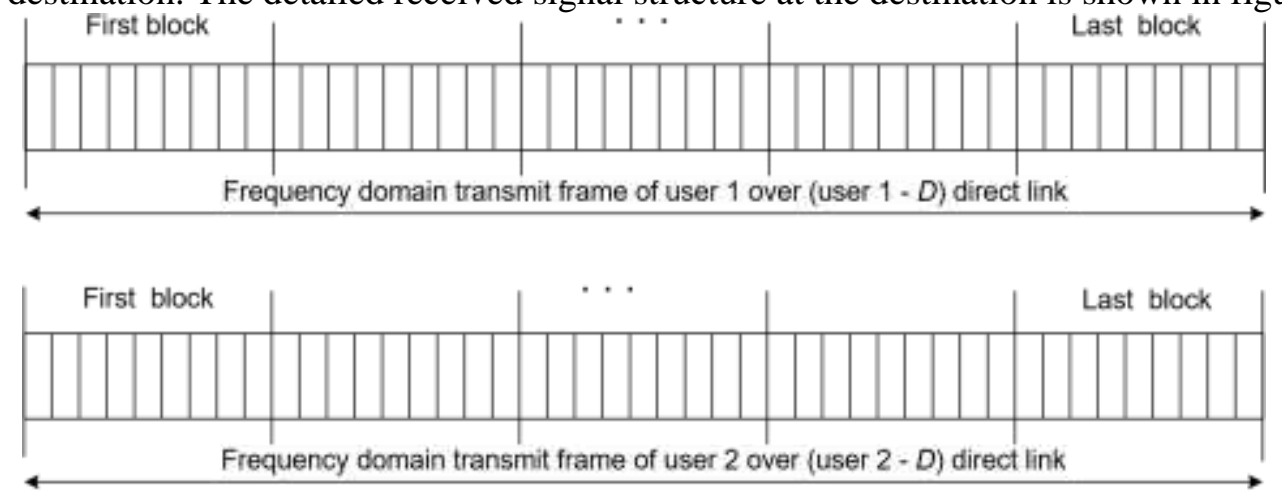


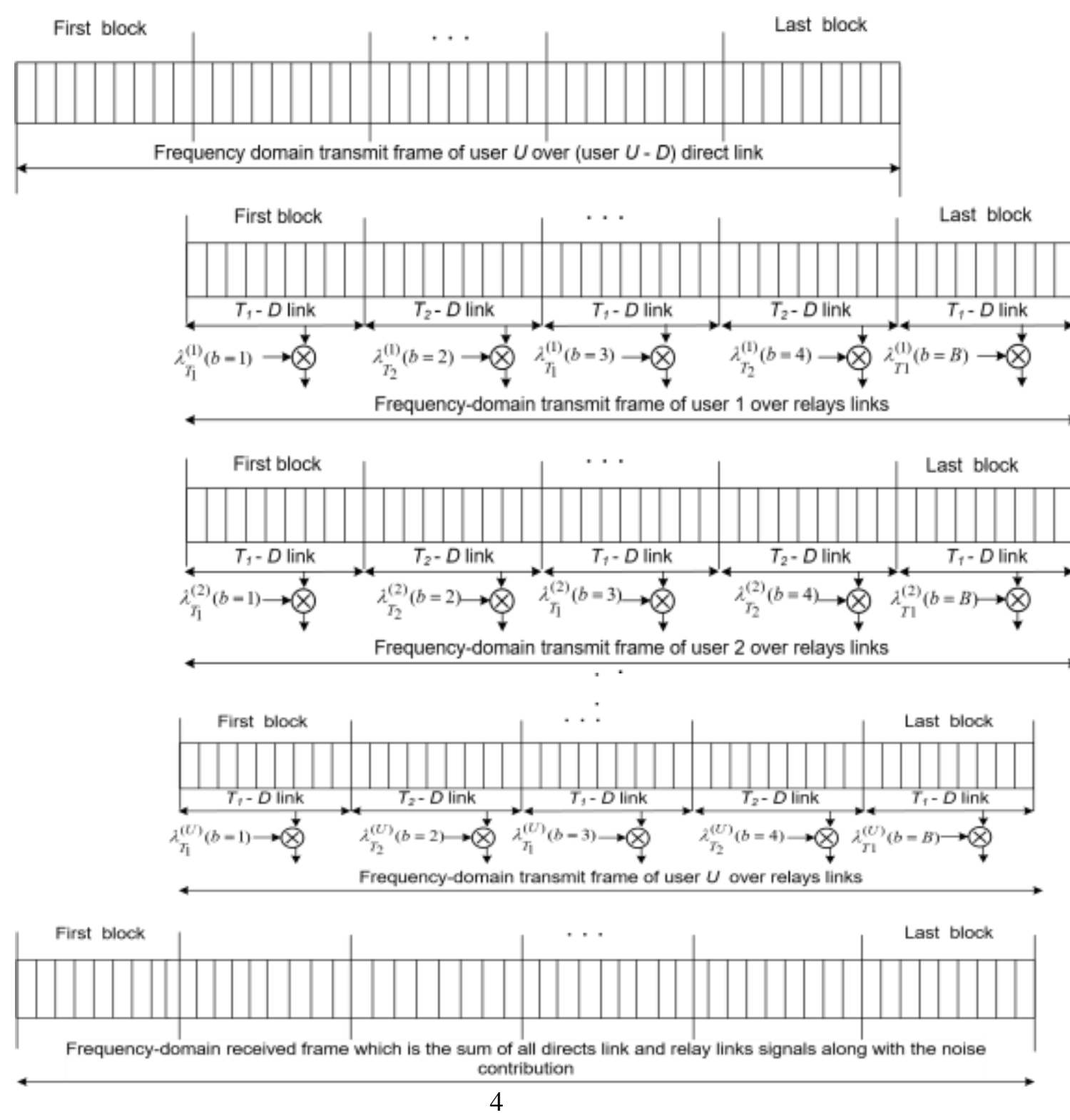

Figure 1. The Detailed Frequency-domain Signal Structure

\section{Proposed First Detector}

At the destination, for each user $u$, the outputs of each block are stacked into a vector $\mathbf{Z}(u)$ as

$$
\begin{gathered}
\mathbb{Z}^{(u)=}[\underbrace{z_{1}^{(u)}(1), z_{1}^{(u)}(2), \cdots, z_{1}^{(u)}(S)}_{\text {first received block }}, \underbrace{z_{2}^{(u)}(1), z_{2}^{(u)}(2), \cdots, z_{2}^{(u)}(S)}_{\text {second received block }} \\
\cdots, \underbrace{z_{B}^{(u)}(1), z_{B}^{(u)}(2), \cdots, z_{B}^{(u)}(S)}_{\text {last received block }}],
\end{gathered}
$$

where $z_{b}{ }^{u)}(?)$ is indicated as in (1).

The first detector is described as follows: choose 
the detected vector $\mathbf{A}^{(i)}=\left\lfloor\hat{\mathbf{a}}_{1}^{(i)}, \cdots, \hat{\mathbf{a}}_{B}^{(i)} \mid\right.$ for which the logarithmic probability density function $\log \operatorname{Pr}\left(\mathbb{Z}^{(i j)} \mid \hat{A}^{(u)}, \mathbf{G}^{(u j}\right)$ is maximum, where $\mathbf{G}^{(u)}$ is a vector having the related

channel coefficients, and $\hat{\mathbf{a}}_{b}{ }^{u}{ }^{u}$ is the corresponding detected symbols of user $u$ which are sent during the block $b$ over sub-carriers $S^{(u)}$. The ML detector of the vector $\mathbf{A}^{(u)}$ can be expressed as

$$
\mathbf{A}^{\wedge}(u)=\underset{\mathbf{A}(u)}{\operatorname{argmax} \log \operatorname{Pr}}\left(\mathbb{Z}^{(u)} \mid \mathbf{A}^{(n)}, \mathrm{G}^{(n)}\right) .
$$

It is noticed from (1) and (2) that independent data streams are send on different subcarriers. This allows us to decouple the overall data detection process into parallel subprocesses. We exploit this fact to develop a simple detector per each sub-carrier. The transmitted symbols on the sub-carrier $s \in S^{(u)}$ is given by

$$
\begin{aligned}
& \quad \underline{\mathbf{A}}^{(u)}(s)=\left[a_{1}^{(u)}(s), a_{2}^{(u)}(s), \cdots, a_{B}^{(u)}(s)\right], \text { and the received symbols are given by } \underline{\mathbf{Z}}^{(u)}(s)= \\
& {\left[z_{1}^{(u)}(s), z_{2}^{(u)}(s), \cdots, z_{B}^{(u)}(s)\right], \text { and }}
\end{aligned}
$$$$
\underline{\mathbf{G}}^{(u)}(s)=\left[\left\{g_{X}^{(u, b)}(s)\right\}_{b=1}^{B},\left\{g_{T_{o}}^{(b)}(s)\right\}_{b=1}^{B},\left\{g_{T_{l}}^{(b)}(s)\right\}_{b=1}^{B}\right]
$$
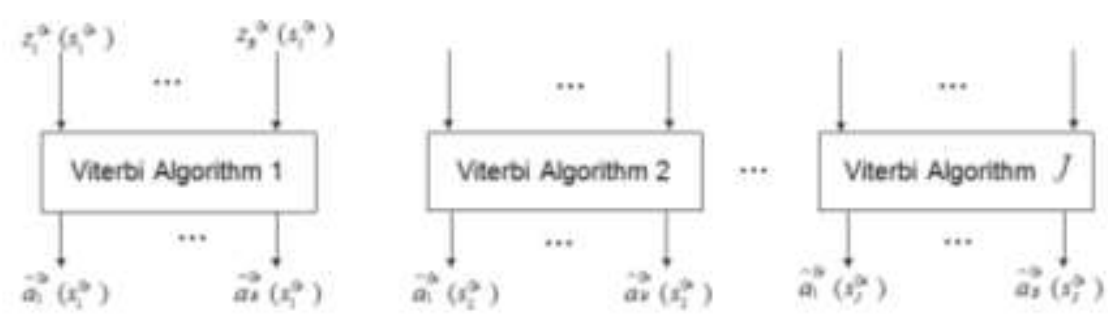

Figure 2. The Conceptual Block Diagram of the First Detector

as the channel coefficients between the user $u$, relays, and the destination. Using the ML principle, we formulate the detection problem related to the sub-carrier $s$ as follows,

$$
\underline{\hat{\mathbf{A}}}^{(u)}(s)=\underset{\underline{\mathbf{A}}^{(u)}(s)}{\arg \max } \log \operatorname{Pr}\left(\underline{\mathbf{Z}}^{(u)}(s) \mid \underline{\mathbf{A}}^{(u)}(s), \underline{\mathbf{G}}^{(u)}(s)\right) .
$$

and it follows that,

$$
\begin{aligned}
& \log \operatorname{Pr}\left(\underline{\mathbf{z}}^{(u)}(s) \mid \underline{\mathbf{A}}^{(u)}(s), \underline{\mathbf{G}}^{(u)}(s)\right)=\sum_{\log \operatorname{Pr}}^{B}\left(z_{b}^{(u)}(s) \mid \underline{\mathbf{a}}_{b}^{(u)}(s), \underline{\boldsymbol{t}}^{(u, b)}(s)\right),
\end{aligned}
$$

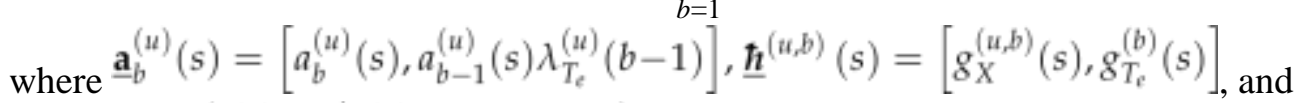

$$
\begin{aligned}
& \operatorname{Pr}\left(z_{b}^{(u)}(s) \mid \underline{\mathbf{a}}_{b}^{(u)}(s), \underline{\hbar}^{(u, b)}(s)\right)= \\
& \text { and } \sigma_{n}^{2} \\
& \frac{1}{\pi \sigma^{2}} \exp \left(-\left|z_{b}^{(u)}(s)-a_{b}^{(u)}(s) g_{X}^{(u, b)}(s)-a_{b-1}^{(u)}(s) \lambda_{T_{e}}^{(u)}(b-1) g_{T_{e}}^{(b)}(s)\right| / \sigma_{n}^{2}\right)
\end{aligned}
$$

is the variance of the white Gaussian noise. Careful examination of (5) reveals that the ML solution of the data detection over the sub-carrier $s$ can be obtained by employing a Viterbi decoding algorithm with a path metric given by (7). The conceptual block diagram of the first detector is shown in figure 2 . 


\section{Practical Aspects}

- Since each sub-carrier carries independent data stream, the data detection process of user $u$ can be carried out by using $J$ Viterbi decoding algorithms, as shown in Figure

2.

- The frequency domain channel coefficient between a relay and destination is set to be zero for the $b$ th block of the user $u$ in case of this block is not correctly decoded by that relay.

- The proposed ML data detection process needs a relatively large processing time and storage memory; this motivates us to develop the following detector.

\section{Proposed Second Detector}

The second detector employs the sliding window concept to tackle the processing time problem in the first detector. The basic idea is to segment the received signal into overlapped windows; each has a width of two packets, as shown in Figure 3. The reason of choosing the width to be two is to maximize the diversity gain; (1 and 2) implies that data symbols of a certain packet are received in two consecutive time slots when a relay is able to correctly detect this packet. From each window we detect data symbols of a packet. Then, we remove the contribution of that packet from the next windows in order to sequentially detect the next packets. Therefore, there is no need to wait the entire frame as occurred in the first detector. The mathematical treatment is provided as follows.

Let us use the first received two packets to detect the sth symbol of the first packet of the $u$ th user. We can write the following

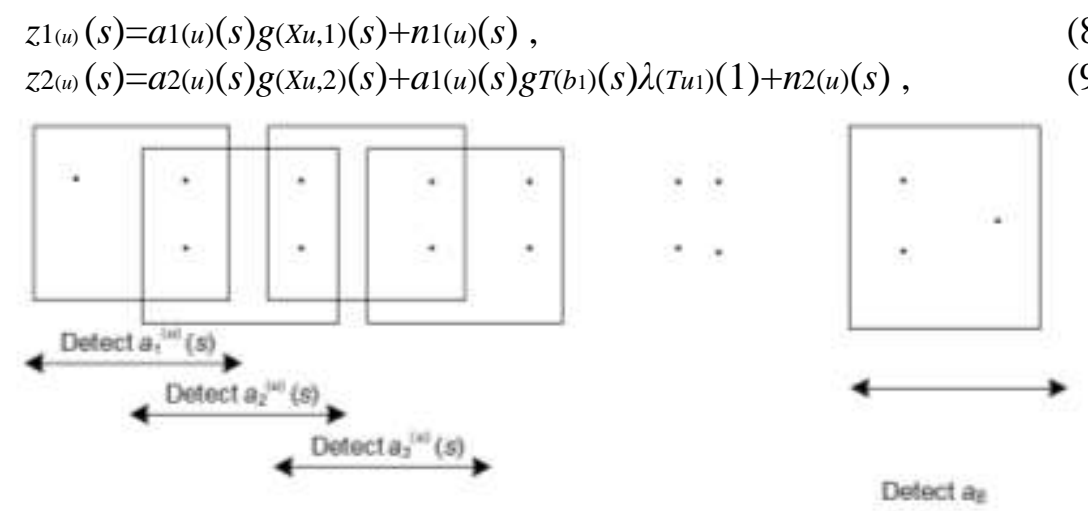

\section{Figure 3. The Conceptual Block Diagram of the Second Detector}

It can be seen from the previous two equations that the detection of $a_{1}{ }^{u}(s)$ is interfered by $a_{2}{ }^{u)}(s)$ which is unknown to the receiver. Therefore, the detection process of $a_{1}{ }^{(u)}(s)$ can be carried out under averaging over all possible values of $a_{2}{ }^{u}(s)$ as follows

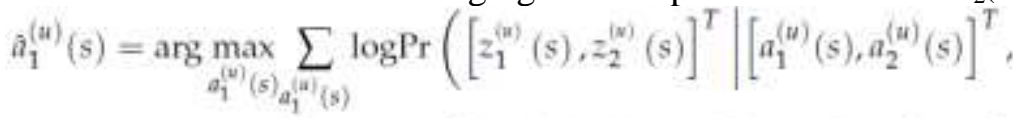

$$
\begin{aligned}
& \left.\left[g_{\mathrm{X}}^{(u, 1)}, g_{\mathrm{X}}^{(u, 2)}(s), g_{T_{1}}^{(b)}(s) \lambda_{T_{1}}^{(u)}(1)\right], \operatorname{Pr}\left(a_{2}^{(u)}(s)\right)\right)
\end{aligned}
$$

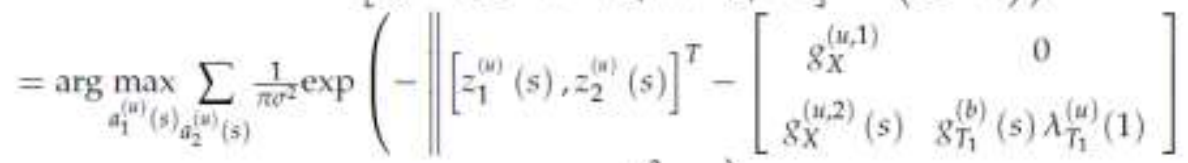

$$
\begin{aligned}
& \left.\left[a_{1}^{(u)}(s), a_{2}^{(i u)}(s)\right]^{T} \|^{2} / \sigma_{n}^{2}\right) \text {. }
\end{aligned}
$$


This process is repeated for $s=s_{1}{ }^{(u)}, s_{2}{ }^{(u)}, \cdots, s_{J}^{(u)}$. After detecting $S$ data symbols of the first packet, its contributions is removed from the received data symbol of the second packet as

$$
\begin{aligned}
\underline{z}_{2}^{(u)}(s) & =z_{2}^{(u)}(s)-a_{1}^{(u)}(s) g_{T_{1}}^{(b)}(s) \lambda_{T_{1}}^{(u)}(1) \\
& =a 2(u)(s)+n 2(u)(s) .
\end{aligned}
$$

Here we follow the common assumption associated with most of the successive detection algorithms found in the literature that the previous symbol is perfectly detected. This assumption is necessary for the theoretical development of the sub-optimal detector; how-

in the simulations, we relax this assumption. Similarly, from $\underline{z}_{2}(s)$ and $z_{3}(s)$ and by averaging over $a_{3}\left({ }^{u)}(s)\right.$, we can detect $a_{2}{ }^{(u)}(s)$. The process is repeated until all data symbols of all packets are detected.

\section{Simulation Results}

Monte Carlo simulations was performed for the validation of the proposed algorithms. Interleaved sub-carrier assignment for multiuser OFDM TPC system was considered. The following parameters were used, $Q=256$ is the number of sub-carriers, $U=4$ is the number of users, and the number of sub-carriers assigned is ${ }_{U}^{Q}=64$ for each user. For each user, a block length of $B=64$ data symbols belonging to 8-PSK constellation was chosen, and the transmission was assumed to be frame based transmission with 20 blocks each, and $L=8$ representing the maximum number of sample-spaced channel taps among all channels. We modeled the channel between nodes $V_{1}$ and node $V_{2}$ as being a zeromean complex Gaussian random variable with an exponential power delay profile:

$$
\sigma_{V_{1} V_{2}}^{2}(l)=\mathrm{Y}_{V_{1} V_{2}} \exp (-l / 5), l=0, \ldots, L_{V_{1} V_{2}}-1
$$

and we selected $L_{V 1} V_{2}$ randomly between the integers of 1 and $L$, and the choice of $Y_{V_{1} V_{2}}$ between users 1-4 and the destination was $1,1.25,0.85$, and 1.1, respectively. For the channel between the two relays and the destination, $\mathrm{Y}_{V_{1} V_{2}}$ was 2.2 and 1.8, respectively.

BER performance for the proposed detectors for different modulation formats is shown in Figure 4, also the performance for the non-interference case is shown as a benchmark, and we can see from the graphs that the proposed detectors match the case of interference cancellation for both detectors.

Performance of the proposed algorithms for different numbers of blocks per frame and different number of sub-carriers $B$ and $Q$ respectively are shown in Figures 5 and 6 , and it can be seen that the performance of both detectors are independent of the values of $B$ and $Q$. 


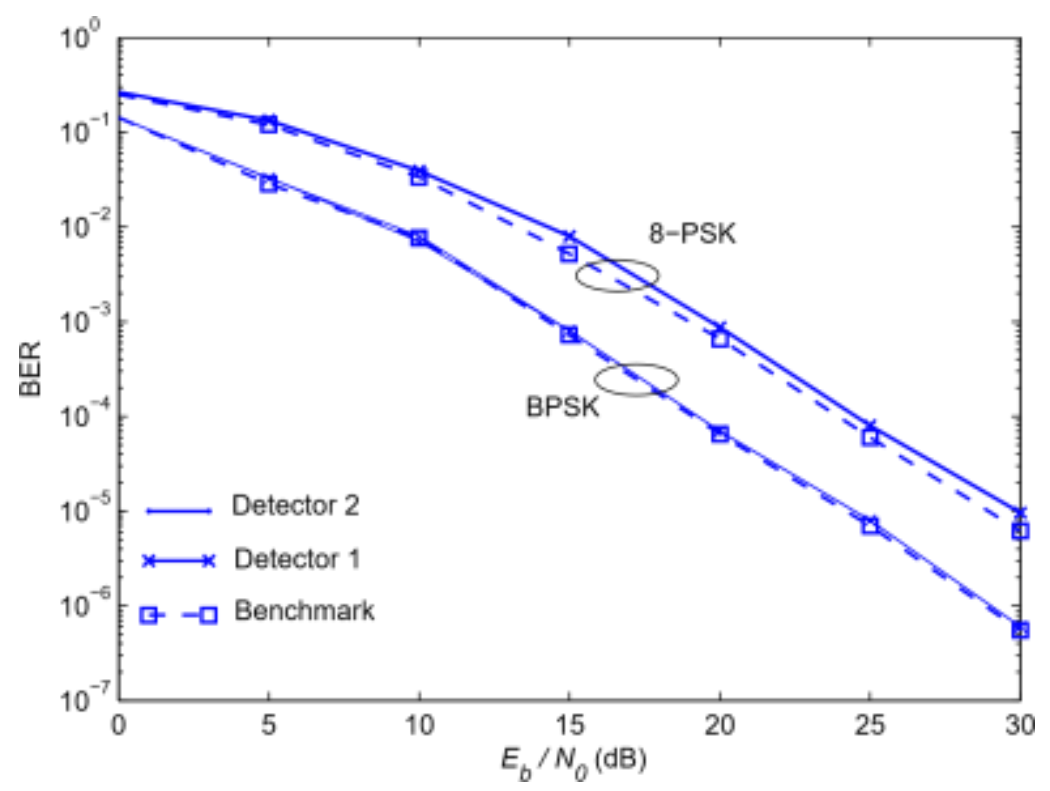

Figure 4. BER Performance of the Proposed Detectors with Different Modulation Formats, BPSK and 8-PSK

\section{Conclusions}

The design of the uplink transmission of the multiuser OFDM TPC system at the destination was investigated. The implemented of a detector using parallel Viterbi algorithms was presented. It was shown that due to the constrain of receiving the entire frame for the proposed detector to operate, gives a limitation factor, mainly delay. Such limitation factor was reduced by proposing the second detector where detecting one block requires receiving two consecutive blocks. Results show that the performance of both detectors are close to each other and to the benchmark of no interference signal at the destination.

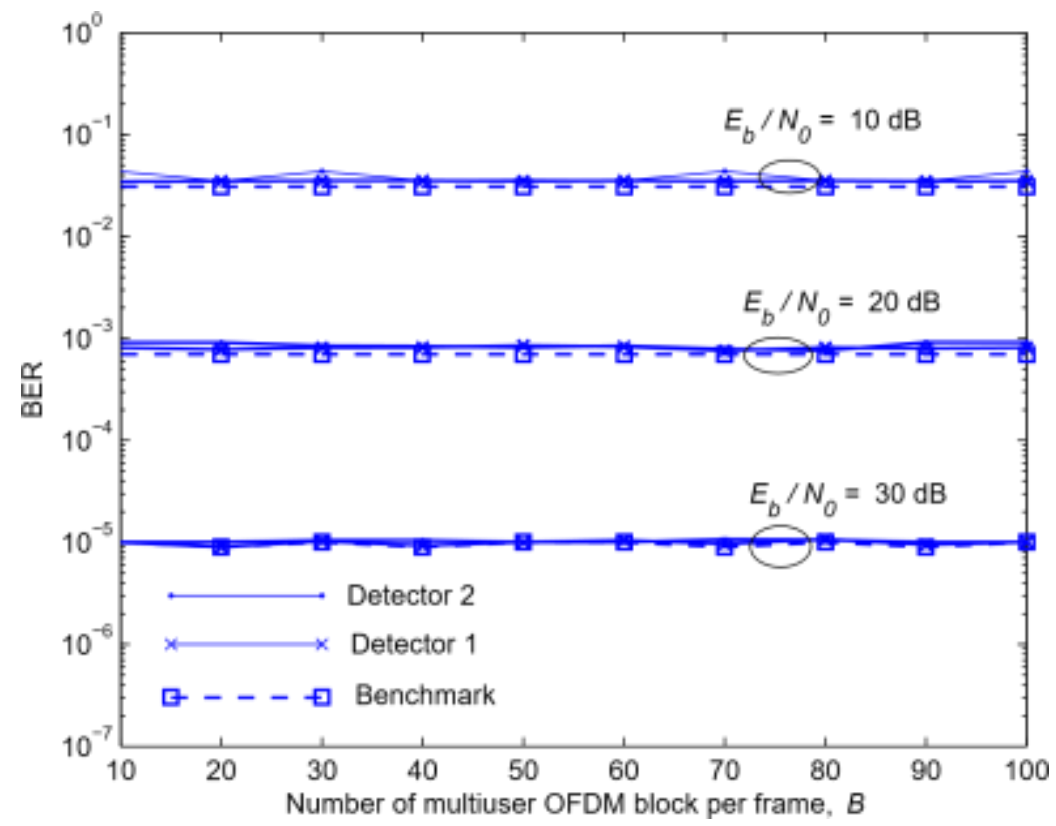

Figure 5. Influence of $B$ on BER for 8-PSK Modulation 


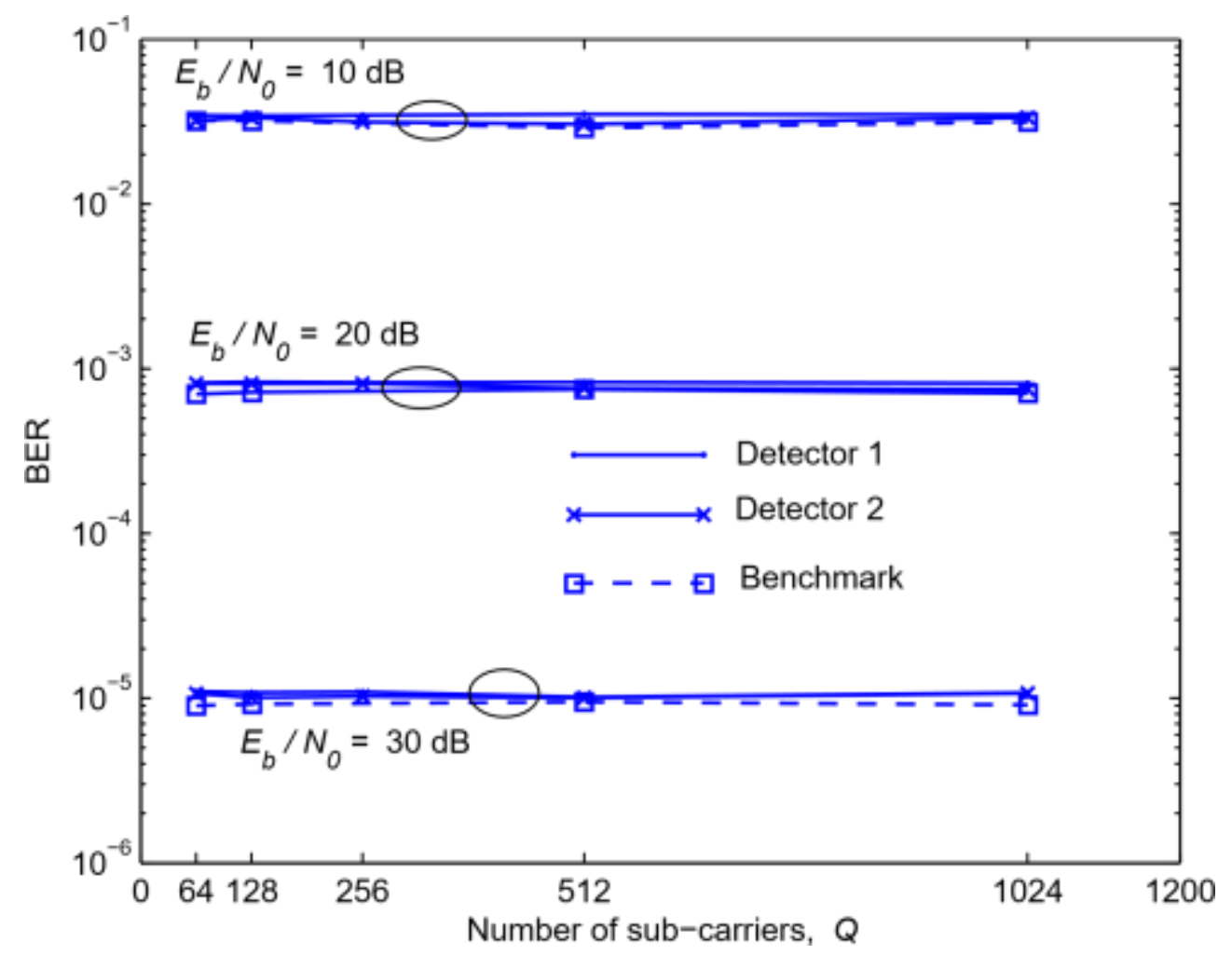

Figure 6. Influence of $Q$ on BER for 8-PSK

\section{References}

[1] C. Wang, Y. Fan, J. Thompson, M. Skoglund, and H. Poor. Approaching the Optimal DiversityMultiplexing Tradeoff in a Four-node Cooperative Network. IEEE Transactions on Wireless Communications, vol. 12, no. 9, (2010), pp. 3690-3700.

[2] H. Mostafa, M. Marey, M. Ahmed, and O. A. Dobre. Simplified Maximum-likelihood Detectors for Fullrate Alternate-relaying Cooperative Systems. IET Communications, vol. 7, no. 17, (2013), pp. 1899 $-1906$.

[3] H. Mostafa, M. Marey, M. H. Ahmed, O. A. Dobre. Decoding Techniques for Alternate-Relaying Cooperative Systems. EURASIP Journal on Wireless Communications and Networking, vol. 1, (2013).

[4] H. Wicaksana, S. Ting, C. Ho, W. Chin, and Y. Guan. AF Two-Path Half Duplex Relaying with InterRelay Self Interference Cancellation: Diversity Analysis and its Improvement. IEEE Transactions on Wireless Communication, vol. 8, no. 9, (2009), pp. 4720-4729.

[5] J. Baek and J. Seo. Efficient iterative SIC and detection for two-path cooperative block transmission relaying. IEEE Communications Letters, vol. 16, no. 2, (2012), pp. 199-201.

[6] M. Marey, H. Mostafa, O. A. Dobre, and M. Ahmed. Data Detection Algorithms for BICM AlternateRelaying Cooperative Systems with Multiple-Antenna Destination. IEEE Transactions on Vehicular Technology, vol. 65, no. 5, (2016), pp. $3802-3807$.

[7] Q. Li, M. Yu, A. Pandharipande, and X. Ge. Outage analysis of cooperative two-path relay channels. IEEE Transactions on Wireless Communications, vol. 15, no. 5, (2016), pp. 3157-3169.

[8] T. Hwang, C. Yang, G. Wu, S. Li, and G. Li. OFDM and Its Wireless Applications: A Survey. IEEE Transactions on Vehicular Technology, vol.58, no. 4, (2009), pp. 1673 - 1694.

[9] Z. Sheng, K. Leung, and Z. Ding. Cooperative Wireless Networks: From Radio to Network Protocol Designs. IEEE Communications Magazine, vol. 49, no. 5, (2011), pp. 64-69. 